\title{
Tracking consensus of general nonlinear multi-agent systems with external disturbances under directed networks
}

DOI:

10.1109/tac.2019.2905512

\section{Document Version}

Accepted author manuscript

Link to publication record in Manchester Research Explorer

Citation for published version (APA):

Wang, H., Ding, Z., \& Yu, X. (2019). Tracking consensus of general nonlinear multi-agent systems with external disturbances under directed networks. I E E E Transactions on Automatic Control.

https://doi.org/10.1109/tac.2019.2905512

\section{Published in:}

I E E E Transactions on Automatic Control

\section{Citing this paper}

Please note that where the full-text provided on Manchester Research Explorer is the Author Accepted Manuscript or Proof version this may differ from the final Published version. If citing, it is advised that you check and use the publisher's definitive version.

\section{General rights}

Copyright and moral rights for the publications made accessible in the Research Explorer are retained by the authors and/or other copyright owners and it is a condition of accessing publications that users recognise and abide by the legal requirements associated with these rights.

\section{Takedown policy}

If you believe that this document breaches copyright please refer to the University of Manchester's Takedown Procedures [http://man.ac.uk/04Y6Bo] or contact uml.scholarlycommunications@manchester.ac.uk providing relevant details, so we can investigate your claim.

\section{OPEN ACCESS}




\title{
Tracking consensus of general nonlinear multi-agent systems with external disturbances under directed networks
}

\author{
He Wang, Wenwu Yu, Senior Member, IEEE, Zhengtao Ding, Senior Member, IEEE, Xinghuo Yu, Fellow, IEEE
}

\begin{abstract}
This paper considers the fully distributed tracking consensus problem for general nonlinear multi-agent systems with a leader whose control input is nonzero and bounded. First, a new class of distributed state observer for the leader is proposed without the knowledge of the upper bound of the leader's input. Then, the situations that followers are affected by disturbances with unknown upper bound or disturbances generated by exosystems are investigated. Specifically, two distributed control protocols based on the distributed state observer, neural networks, and adaptive laws are proposed. Finally, simulation examples are provided to illustrate the theoretical results.
\end{abstract}

Index terms - tracking consensus, general nonlinearity, external disturbances, unknown upper bound.

\section{INTRODUCTION}

In the past few decades, collective behaviors of multi-agent systems have been ascending into a hot issue because of its widespread application in many fields, such as robotic teams [1], satellite clusters [2], unmanned air vehicles [3], distributed sensor networks [4], and so on. The focal point of the study on the collective behaviors of multi-agent systems lies in analyzing the essence that local information interaction among agents leads to globally collective behaviors.

Consensus problem of multi-agent systems is one of the most widely studied topics about collective behaviors of multiagent systems [5]-[23]. Consensus problem means that, for a group of agents, design some proper distributed control protocols for each agent relying only on local information of the agent and its neighbors, so that these agents can reach an agreement. Generally, consensus problem can be divided into two categories, one is the leaderless consensus problem (without a leader) [5] [13] [14], the other is the leader-follower consensus (with one or more leaders) problem [30]-[34]. One generally studied model in multi-agent systems is the integral chain systems. [5] and [22] considered the consensus and bipartite consensus problems of first-order multi-agent systems

This work was supported by the National Natural Science Foundation of China under Grant No. 61673107, the National Ten Thousand Talent Program for Young Top-notch Talents under Grant No. W2070082, the General joint fund of the equipment advance research program of Ministry of Education under Grant No. 6141A020223, the Jiangsu Provincial Key Laboratory of Networked Collective Intelligence under Grant No. BM2017002, and the Scientific Research Foundation of Graduate School of Southeast University under Grant No. YBJJ1717.

H. Wang and W. Yu are with the School of Mathematics, Southeast University, Nanjing 210096, China (e-mail: wanghe@ seu.edu.cn; wwyu@ seu.edu.cn) (corresponding author: Wenwu $\mathrm{Yu}$ ).

Z. Ding is with the School of Electrical and Electronic Engineering, University of Manchester, Sackville Street Building, Manchester M13 9PL, UK (email: zhengtao.ding@manchester.ac.uk).

$\mathrm{X}$. Yu is with the School of Engineering, RMIT University, Melbourne VIC 3001, Australia (e-mail: x.yu@ rmit.edu.au). with both undirected and directed communication topologies, respectively. [9] analyzed the tracking consensus problem for first-order multi-agent systems. [19] and [20] further considered the finite-time and fixed-time consensus problems for first-order multi-agent systems, respectively. [13] provided some necessary and sufficient conditions for achieving secondorder asymptotical consensus. A further consideration on the consensus problem of second-order nonlinear multi-agent systems was given in [14]. [15]-[17] considered the finite-time consensus and tracking consensus problems for second-order multi-agent systems, respectively. One can refer to [24]-[27] for more consensus results about integral chain multi-agent systems.

Another extensively researched model for multi-agent systems is the general linear time-invariant systems. During the last decade, plentiful works about the consensus problem of linear multi-agent systems have been reported [28]-[35]. [30] and [31] studied the fully distributed tracking consensus problem for a class of linear multi-agent systems under undirected and directed graphs, respectively. In these two works, the leader was assumed having no input. The situation that the leader has bounded input under general directed graphs was further investigated in [32] and [33]. The relative state and output information were utilized in designing fully distributed control protocols in [32] and [33], respectively. [35] considered the distributed containment control problem for a class of linear multi-agent systems with more than one leader under directed graphs. However, in most real control systems, nonlinearity is ubiquitous. In view of this, the consensus problem of general nonlinear multi-agent systems was discussed in [36][39]. [36] and [37] investigated the tracking consensus problem of general nonlinear multi-agent systems under undirected and general directed graphs, respectively. Both [36] and [37] assumed that the leader had bounded input, and the nonlinearity existed in followers. Neural network was introduced to estimate and counteract nonlinearities, which resulted in that the tracking errors were uniformly ultimately bounded. While in [38] and [39], both the leader and the followers could have Lipschitz-type nonlinearities and the leader had no input, and no disturbance existed.

As discussed above, there are few works concerning about the tracking consensus problem of general nonlinear multiagent systems that the leader could have unknown bounded input, and the followers have general nonlinear dynamics and could be affected by external disturbances. The difficulty of such kind problems mainly lies in the coupling between external disturbances and nonlinearity, and the unknown upper bound of leader's input and disturbances. The main contributions of this work are two-fold. First, a fully distributed state observer for the leader under general directed communication topologies is addressed, where the upper bound of 
the leader's input is unknown to all the followers. To the best of the authors' knowledge, there are very few works having solved such kind of problem. Second, for each follower with disturbances (disturbances with unknown upper bound or disturbances generated by exosystems) and nonlinearity, neural networks are introduced to estimate and compensate the nonlinearity. Even the disturbances and nonlinearity are coupled together, the tracking problem can still be solved with the help of the proposed distributed state observer.

The rest of this paper is arranged as follows. In Section II, some preliminaries and the tracking problem are introduced. In Section III, a new class of distributed state observer for the leader is proposed. The tracking consensus problem with bounded disturbances and disturbances generated by exosystems are studied in Section IV and V, respectively. Some simulations are presented to verify the theoretical results in Section VI, and Section VII concludes this paper.

Notations: $\lambda_{\min }(E)\left(\lambda_{\max }(E)\right)$ means the minimal (maximal) eigenvalue of the symmetric real matrix $E .\|\cdot\|$ denotes the Euclidean norm and $\|\cdot\|_{1}$ denotes the 1-norm. For any matrix $\mathcal{A} \in R^{m \times n}$, let $\|\mathcal{A}\|_{F}=\sqrt{\operatorname{tr}\left(\mathcal{A}^{T} \mathcal{A}\right)}$, where $\operatorname{tr}(\cdot)$ denotes the trace of a square matrix. Besides, for a vector $x=\left[x_{1}, \ldots, x_{n}\right]^{T} \in R^{n}$, denote $\|x\|_{\infty}=\max _{i=1, \ldots, N}\left\{\left|x_{i}\right|\right\}$. Furthermore, $1_{N}$ means an $N$-dimensional column vector with all elements being 1.0 is used to represent the matrix block of appropriate dimensions with all elements being 0 . Denote $\operatorname{sym}(H)=H+H^{T}$ with $H$ being a square matrix. For a real symmetric matrix $E, E>0$ means that $E$ is positive definite. sign function is defined as: $\operatorname{sign}(x)=1$, if $x>0$; $\operatorname{sign}(x)=-1$, if $x<0$; and $\operatorname{sign}(0)=0$.

\section{PREliminaries AND Model Description}

\section{A. Preliminaries}

In this subsection, some basic concepts about graph theory and some useful lemmas are introduced.

A directed graph (or digraph) $\mathcal{G}=(\mathcal{V}, \mathcal{E}, G)$ involves a set of nodes which is defined by $\mathcal{V}=\left\{v_{1}, v_{2}, \ldots, v_{N}\right\}$, a set of edges $\mathcal{E} \subseteq \mathcal{V} \times \mathcal{V}$ and a weighted adjacency matrix $G=\left(a_{i j}\right)_{N \times N}$. Here, an edge $\mathcal{E}_{i j}$ in $\mathcal{G}$ means a pair of nodes $\left(v_{i}, v_{j}\right)$ representing that the information flow goes from node $v_{j}$ to node $v_{i}$. $G$ records the coupling configuration information of all the edges in $\mathcal{G}$. The weight $a_{i j}$ is defined as: $a_{i j}>0 \Leftrightarrow\left(v_{i}, v_{j}\right) \in \mathcal{E}$. In this paper, self loop is not considered, i.e. $a_{i i}=0, i=1,2, \ldots, N$.

Definition 1: [6][14] A directed path from node $v_{j}$ to $v_{i}$ is a sequence of directed edges $\left(v_{i}, v_{i_{1}}\right),\left(v_{i_{1}}, v_{i_{2}}\right), \ldots,\left(v_{i_{l}}, v_{j}\right)$ in the directed network with distinct nodes $v_{i_{k}}, k=1,2, \ldots, l$. A directed network $\mathcal{G}$ is strongly connected if between any pair of distinct nodes $v_{i}$ and $v_{j}$ in $\mathcal{G}$, there exists a directed path from $v_{i}$ to $v_{j}, i, j=1,2, \ldots, N$. A directed network $\mathcal{G}$ is said to contain a directed spanning tree, if there exists a node named root that, this node has directed paths to all the other nodes of this network $\mathcal{G}$.

The Laplacian matrix $L=\left(l_{i j}\right)_{N \times N}$ for a directed graph $\mathcal{G}$ is denoted by [6]:

$$
l_{i i}=\sum_{j=1, j \neq i}^{N} a_{i j} ; \quad l_{i j}=-a_{i j}, i \neq j .
$$

Lemma 1: [41] For any matrices $\mathcal{A} \in R^{m \times n}$ and $\mathcal{B} \in$ $R^{n \times m}$, it holds that $|\operatorname{tr}(\mathcal{A B})| \leq\|\mathcal{A}\|_{F} \cdot\|\mathcal{B}\|_{F}$. Besides, $\|\mathcal{A}\| \leq\|\mathcal{A}\|_{F}$.

\section{B. Model Description}

In this subsection, the tracking problem will be formulated. Consider a group of $N+1$ agents with unknown heterogeneous nonlinear dynamics, consisting of one leader and $N$ followers. Suppose that each agent has the following general nonlinear dynamics:

$$
\begin{gathered}
\dot{x}_{0}(t)=A x_{0}(t)+B u_{0}(t), \\
\dot{x}_{i}(t)=A x_{i}(t)+B\left[u_{i}(t)+f_{i}\left(x_{i}(t)\right)+d_{i}(t)\right], \\
i=1,2, \ldots, N,
\end{gathered}
$$

where $x_{0}(t) \in R^{n}$ and $u_{0}(t) \in R^{m}$ represent the leader's state and control input, $x_{i}(t) \in R^{n}, u_{i}(t) \in R^{m}, f_{i}: R^{n} \rightarrow R^{m}$, and $d_{i}(t) \in R^{m}$ represent the state, the control input, the heterogeneous smooth nonlinearity, and the disturbance of the $i$ th follower, respectively, $i=1, \ldots, N . A \in R^{n \times n}, B \in R^{n \times m}$ are constant matrices. In the following, $x_{i}$ and $u_{i}$ are used for simplification.

Definition 2: The tracking consensus is said to be achieved if and only if there exist some proper control inputs $u_{i}, i=$ $1,2, \ldots, N$, such that:

$$
\lim _{t \rightarrow \infty}\left\|x_{i}-x_{0}\right\|=0, i=1, \ldots, N .
$$

\section{A CLASS OF FULLY DISTRIBUTED STATE OBSERVER FOR THE LEADER}

In this section, a new class of fully distributed observer will be constructed to estimate the state of the leader. First of all, some assumptions about the dynamics of the leader and the communication network among the agents are given as follows.

Assumption 1: The pair $(A, B)$ is controllable.

Lemma 2: [47][40] With Assumption $1, \forall \bar{\beta}>0$, there exists a solution $P>0$ to the following Riccati inequality

$$
P A+A^{T} P-2 \bar{\beta} P B B^{T} P+\bar{\beta} I_{n}<0 .
$$

Assumption 2: There exists a constant $\omega>0$ such that $\left\|u_{0}\right\| \leq \omega$. Besides, the upper bound $\omega$ is unknown to all the followers.

Assumption 3: The state of the leader is bounded.

Remark 1: The leader could be a real agent or a vitual signal with predefined trajectory so as to render the multi-agent system to fulfill specific control objectives. In real applications, the workspace is usually with limited space, hence it could be reasonable to ensure that the leader has bounded state (by designing $u_{0}$ in advance). However, each follower still has no knowledge about $u_{0}$.

Assumption 4: The communication network of the $N+1$ agents contains a directed spanning tree with the leader as the root node.

Let $L_{f}$ be the Laplacian matrix corresponding to the subgraph among all the followers, and denote $\Psi=$ $\operatorname{diag}\left(a_{10}, \ldots, a_{N 0}\right)$. Define $\bar{L}=L_{f}+\Psi$. The following lemma provides an useful property of $\bar{L}$.

Lemma 3: [7] Suppose that Assumption 4 is satisfied, then there exists a diagonal matrix $\Xi$ such that $\Xi=$ $\operatorname{diag}\left(\xi_{1}, \ldots, \xi_{N}\right)>0$ and $\Xi \bar{L}+\bar{L}^{T} \Xi>0$.

Before showing the distributed state obsever design, the structure of the dynamics of the leader needs to be further analyzed.

Definition 3: [42] The pairs $(A, B)$ and $\left(A^{\prime}, B^{\prime}\right)$ are said feedback equivalent (or F-equivalent) iff there exist matrices $C \in R^{m \times m}, D \in R^{n \times n}$, and $Q \in R^{m \times n}$, with $C$ and $D$ being nonsingular, such that $A^{\prime}=C^{-1}(A+B Q) C$, and $B^{\prime}=$ $C^{-1} B D$. 
Lemma 4: [42] Suppose that $(A, B)$ is controllable. Denote $r_{0}=\operatorname{rank}(B)$. Then, there exist $m$ integers $p_{1}, \ldots, p_{m}$, satisfying $0 \leq p_{i} \leq n, p_{1} \geq p_{2} \geq \ldots \geq p_{r_{0}}>0, p_{i}=0$, for $i>r_{0}$, and $\sum_{i=1}^{m} p_{i}=n$, such that $(A, B)$ is F-equivalent with a decoupled system of $r_{0}$ integrators:

$$
\begin{gathered}
\dot{y}_{k_{i}+1}=y_{k_{i}+2}, \ldots, \dot{y}_{k_{i+1}-1}=y_{k_{i+1}}, \dot{y}_{k_{i+1}}=v_{i+1}, \\
i=0, \ldots, r_{0}-1, k_{0}=0, k_{s}=\sum_{j=1}^{s} p_{j}, s=1, \ldots, r_{0} .
\end{gathered}
$$

According to Lemma 4, there exist matrices $C, D$, and $Q$, with the state transformation $y_{0}=C^{-1} x_{0}$, such that

$$
\dot{y}_{0}=A^{\prime} y_{0}+B^{\prime} u_{y_{0}}
$$

has the form

$$
\begin{gathered}
\dot{y}_{0, k_{l}+1}=y_{0, k_{l}+2}, \ldots, \dot{y}_{0, k_{l+1}-1}=y_{0, k_{l+1}}, \dot{y}_{0, k_{l+1}}=d_{0, l+1}, \\
l=0, \ldots, r_{0}-1, k_{0}=0, k_{s}=\sum_{j=1}^{s} p_{j}, s=1, \ldots, r_{0},
\end{gathered}
$$

where $y_{0}=\left[y_{01}, \ldots, y_{0 n}\right]^{T}, A^{\prime}=C^{-1}(A+B Q) C$, $B^{\prime}=C^{-1} B D, u_{y_{0}}=D^{-1}\left(-Q x_{0}+u_{0}\right)$, and $B^{\prime} u_{y_{0}}=$ $\left[0_{1 \times p_{1}-1}, d_{01}, 0_{1 \times p_{2}-1}, d_{02}, \ldots, 0_{1 \times p_{r_{0}}-1}, d_{0 r_{0}}\right]^{T}$. With Assumptions 2 and 3 , one has $d_{01}, \ldots, d_{0 r_{0}}$ are all bounded, and the upper bounds are unknown to all the followers. Suppose that $\left|d_{0 l}\right| \leq D_{0}, l=1, \ldots, r_{0}$, with $D_{0}$ being a finite positive number.

To this end, it is ready to present the construction of the fully distributed state observer. Specifically, for follower $i$, consider the following distributed state observer:

$$
\begin{aligned}
& \dot{y}_{i, k_{l}+1}=y_{i, k_{l}+2}-e_{i, k_{l}+1}, \\
& \ldots \\
& \dot{y}_{i, k_{l+1}-1}=y_{i, k_{l+1}}-e_{i, k_{l+1}-1}, \\
& \dot{y}_{i, k_{l+1}}=-\left(\alpha_{i, l+1}+\beta_{i, l+1}\right)\left[e_{i, k_{l+1}}+\operatorname{sign}\left(e_{i, k_{l+1}}\right)\right], \\
& l=0, \ldots, r_{0}-1, k_{0}=0, k_{s}=\sum_{j=1}^{s} p_{j}, s=1, \ldots, r_{0},
\end{aligned}
$$

where $e_{i k}=\sum_{j=1}^{N} a_{i j}\left(y_{i k}-y_{j k}\right)+a_{i 0}\left(y_{i k}-y_{0 k}\right), k=1, \ldots, n$, $\beta_{i, l+1}=\frac{1}{2} e_{i, k_{l+1}}^{2}+\left|e_{i, k_{l+1}}\right|$, and $\alpha_{i, l+1}$ is an adaptive control gain designed as follows:

$$
\begin{aligned}
& \dot{\alpha}_{i, l+1}=\left|e_{i, k_{l+1}}+\operatorname{sign}\left(e_{i, k_{l+1}}\right)\right|, \\
& \alpha_{i, l+1}(0)>0, \quad l=0, \ldots, r_{0}-1 .
\end{aligned}
$$

Theorem 1: Suppose that Assumptions 1-4 are satisfied. Then the distributed state observer (6) and (7) can estimate the state of the leader asymptotically.

Proof: Observing that in (6), the $r_{0}$ integrator-like components are decoupled from each other, one only needs to prove that each component in (6) can cooperatively estimate the states of the corresponding integrator in (5). Without loss of generality, only the first component (first $p_{1}$ rows) of (6) will be analyzed in the following.

Denote $e_{k}=\left[e_{1 k}, \ldots, e_{N k}\right]^{T}, \alpha_{1}=\operatorname{diag}\left(\alpha_{11}, \ldots, \alpha_{N 1}\right)$, $\beta_{1}=\operatorname{diag}\left(\beta_{11}, \ldots, \beta_{N 1}\right)$. Then one can obtain the dynamics of $\left[e_{1}^{T}, \ldots, e_{p_{1}}^{T}\right]^{T}$ by subtracting (5) from (6):

$$
\begin{aligned}
\dot{e}_{1} & =e_{2}-\bar{L} e_{1} \\
& \ldots \\
\dot{e}_{p_{1}-1} & =e_{p_{1}}-\bar{L} e_{p_{1}-1} \\
\dot{e}_{p_{1}} & =-\bar{L}\left(\alpha_{1}+\beta_{1}\right)\left(e_{p_{1}}+\operatorname{sign}\left(e_{p_{1}}\right)\right)-\bar{L} 1_{N} d_{01} .
\end{aligned}
$$

Consider the following Lyapunov function

$$
V_{o}=\sum_{i=1}^{N} \xi_{i} \alpha_{i 1} \beta_{i 1}+\frac{1}{2} \sum_{i=1}^{N} \xi_{i}\left[\beta_{i 1}^{2}+\left(\alpha_{i 1}-\bar{\alpha}\right)^{2}\right],
$$

where $\xi_{i}$ is defined in Lemma 3, and $\bar{\alpha}$ is a positive constant to be designed.

Taking the derivative of $V_{o}$, one gets

$$
\begin{aligned}
\dot{V}_{o}= & \sum_{i=1}^{N} \xi_{i}\left(\alpha_{i 1}+\beta_{i 1}\right) \dot{\beta}_{i 1}+\sum_{i=1}^{N} \xi_{i} \beta_{i 1} \dot{\alpha}_{i 1}+\sum_{i=1}^{N} \xi_{i}\left(\alpha_{i 1}-\bar{\alpha}\right) \dot{\alpha}_{i 1} \\
= & 1_{N}^{T}\left(\alpha_{1}+\beta_{1}\right) \Xi \operatorname{diag}\left(e_{p_{1}}+\operatorname{sign}\left(e_{p_{1}}\right)\right) \cdot \\
& {\left[-\bar{L}\left(\alpha_{1}+\beta_{1}\right)\left(e_{p_{1}}+\operatorname{sign}\left(e_{p_{1}}\right)\right)-\bar{L} 1_{N} d_{01}\right] } \\
& +\sum_{i=1}^{N} \xi_{i}\left(\alpha_{i 1}+\beta_{i 1}\right) \dot{\alpha}_{i 1}-\bar{\alpha} \sum_{i=1}^{N} \xi_{i} \dot{\alpha}_{i 1} \\
\leq & -\lambda_{0}\left(e_{p_{1}}+\operatorname{sign}\left(e_{p_{1}}\right)\right)^{T}\left(\alpha_{1}+\beta_{1}\right)^{2}\left(e_{p_{1}}+\operatorname{sign}\left(e_{p_{1}}\right)\right) \\
& +\sum_{i=1}^{N} D_{0} a_{i 0} \xi_{i}\left(\alpha_{i 1}+\beta_{i 1}\right)\left|e_{i p_{1}}+\operatorname{sign}\left(e_{i p_{1}}\right)\right| \\
& +\sum_{i=1}^{N} \xi_{i}\left(\alpha_{i 1}+\beta_{i 1}\right)\left|e_{i p_{1}}+\operatorname{sign}\left(e_{i p_{1}}\right)\right| \\
& -\bar{\alpha} \sum_{i=1}^{N} \xi_{i}\left|e_{i p_{1}}+\operatorname{sign}\left(e_{i p_{1}}\right)\right| \\
= & -\lambda_{0} \sum_{i=1}^{N}\left(\alpha_{i 1}+\beta_{i 1}\right)^{2}\left|e_{i p_{1}}+\operatorname{sign}\left(e_{i p_{1}}\right)\right|^{2} \\
& +\sum_{i=1}^{N} \xi_{i}\left(D_{0} a_{i 0}+1\right)\left(\alpha_{i 1}+\beta_{i 1}\right)\left|e_{i p_{1}}+\operatorname{sign}\left(e_{i p_{1}}\right)\right| \\
& -\bar{\alpha} \sum_{i=1}^{N} \xi_{i}\left|e_{i p_{1}}+\operatorname{sign}\left(e_{i p_{1}}\right)\right|, \\
& \lambda(10)
\end{aligned}
$$

where $\lambda_{0}=\lambda_{\min }\left(\frac{1}{2}\left(\bar{L}^{T} \Xi+\Xi \bar{L}\right)\right)$, and the facts that $1_{N}^{T}\left(\alpha_{1}+\right.$ $\left.\beta_{1}\right) \Xi \operatorname{diag}\left(e_{p_{1}}+\operatorname{sign}\left(e_{p_{1}}\right)\right)=\left(e_{p_{1}}+\operatorname{sign}\left(e_{p_{1}}\right)\right)^{T}\left(\alpha_{1}+\beta_{1}\right) \Xi$ , $\bar{L} 1_{N}=\left[a_{10}, \ldots, a_{N 0}\right]^{T}$, and the boundedness of $d_{01}$ are applied.

Choose $\bar{\alpha}$ such that $\bar{\alpha} \geq \max _{i=1, \ldots, N}\left\{\frac{\xi_{i}}{2 \lambda_{0}}\left(D_{0} a_{i 0}+1\right)^{2}\right\}$. Noticing that $\left|e_{i p_{1}}+\operatorname{sign}\left(e_{i p_{1}}\right)\right| \geq 1$ whenever $\left|e_{i p_{1}}\right|>0$, it follows that $\left|e_{i p_{1}}+\operatorname{sign}\left(e_{i p_{1}}\right)\right|^{\frac{3}{2}} \geq\left|e_{i p_{1}}+\operatorname{sign}\left(e_{i p_{1}}\right)\right|$. In view of this property as well as invoking the basic inequality, one has

$$
\begin{aligned}
\dot{V}_{o} \leq & -\frac{\lambda_{0}}{2} \sum_{i=1}^{N}\left(\alpha_{i 1}+\beta_{i 1}\right)^{2}\left|e_{i p_{1}}+\operatorname{sign}\left(e_{i p_{1}}\right)\right|^{2} \\
& -\sum_{i=1}^{N} \sqrt{2 \lambda_{0} \bar{\alpha} \xi_{i}}\left(\alpha_{i 1}+\beta_{i 1}\right)\left|e_{i p_{1}}+\operatorname{sign}\left(e_{i p_{1}}\right)\right|^{\frac{3}{2}} \\
& +\sum_{i=1}^{N} \xi_{i}\left(D_{0} a_{i 0}+1\right)\left(\alpha_{i 1}+\beta_{i 1}\right)\left|e_{i p_{1}}+\operatorname{sign}\left(e_{i p_{1}}\right)\right| \\
& \leq-\frac{\lambda_{0}}{2} \sum_{i=1}^{N}\left(\alpha_{i 1}+\beta_{i 1}\right)^{2}\left|e_{i p_{1}}+\operatorname{sign}\left(e_{i p_{1}}\right)\right|^{2} \\
& \leq-\frac{\lambda_{0}}{2} \sum_{i=1}^{N} \alpha_{i 1}(0)\left|\operatorname{sign}\left(e_{i p_{1}}\right)\right|^{2}
\end{aligned}
$$


where the last inequality is resulted by the facts that 1) $\alpha_{i 1}(t)$ is nondecreasing; 2) $\left.\beta_{i 1} \geq 0 ; 3\right)\left|e_{i p_{1}}+\operatorname{sign}\left(e_{i p_{1}}\right)\right|^{2}=e_{i p_{1}}^{2}+$ $2\left|e_{i p_{1}}\right|+\left|\operatorname{sign}\left(e_{i p_{1}}\right)\right|^{2} \geq\left|\operatorname{sign}\left(e_{i p_{1}}\right)\right|^{2}$.

As long as the tracking consensus is not achieved, there must exist $j \in\{1, \ldots, N\}$, such that $e_{j p_{1}} \neq 0$. Here, $j$ could vary from time. Denote $\alpha_{o}=\frac{\lambda_{0}}{2} \min _{i=1, \ldots, N}\left\{\alpha_{i 1}(0)\right\}$. Then, before the tracking consensus is achieved, one has

$$
\dot{V}_{o} \leq-\alpha_{o}
$$

which follows that $e_{i p_{1}}, i=1, \ldots, N$, would reach to zero no later than $T_{o}=\frac{V_{o}(0)}{\alpha_{o}}$, which means that $y_{i p_{1}}$ can estimate the real value of $y_{0 p_{1}}$ as $t \rightarrow \infty$.

On the other hand, since $-\bar{L}$ is Hurwitz, one can observe from (8) that, the system of $e_{p_{1}-1}$ is input-to-state stable (ISS) with respect to $e_{p_{1}}$. Then, one has $e_{p_{1}-1} \rightarrow 0$ as $e_{p_{1}} \rightarrow 0$, which means that $y_{i, p_{1}-1} \rightarrow y_{0, p_{1}-1}$ as $t \rightarrow \infty$. With similar arguments, one can conclude that $y_{i k} \rightarrow y_{0 k}, k=1, \ldots, p_{1}$, as $t \rightarrow \infty$. This completes the proof.

Remark 2: With the differential inequality (12), one can only claim that the tracking error $y_{i p_{1}}-y_{0 p_{1}}$ will reach zero asymptotically. In fact, the evolution of each follower depends on how $d_{01}$ evolves. For one thing, $V_{o}$ is possible to stop decreasing before reaching to zero if the tracking error $y_{i p_{1}}-y_{0 p_{1}}$ has reached zero before $T_{o}$. This statement can be observed from the discussion of $\dot{V}_{o}$. For another, if $d_{01}$ evolves very slowly and its absolute value reaches maximum after a long time. While $\alpha_{i 1}$, the main component of the coefficient of $\operatorname{sign}\left(e_{i p_{1}}\right)$, increases quickly. Then $\left(\alpha_{i 1}+\beta_{i 1}\right) \operatorname{sign}\left(e_{i p_{1}}\right)$ can dominate the effect caused by $d_{01}$. During this time interval, the tracking error will reach zero fast. When $d_{01}$ evolves above the domination of $\left(\alpha_{i 1}+\beta_{i 1}\right) \operatorname{sign}\left(e_{i p_{1}}\right), \alpha_{i 1}$ increases quickly and makes the followers track the leader. So (12) should be understood as that the distributed observer (6) and (7) has a fast response with respect to the leader's evolution.

Remark 3: Theoretically, $\alpha_{i, l+1}$ is bounded. While it is not the truth in real applications. To ensure the boundedness of adaptive gains in practical implementations, one can use a sufficiently small value $\iota$ to adjust the ideal adaptive law to practical one:

$$
\dot{\alpha}_{i, l+1}= \begin{cases}\left|e_{i, k_{l+1}}+\operatorname{sign}\left(e_{i, k_{l+1}}\right)\right|, & \left|e_{i, k_{l+1}}\right| \geq \iota, \\ 0, & \left|\hat{e}_{v i}\right|<\iota .\end{cases}
$$

Remark 4: One should notice that if the leader is originally a higher-order integrator, then the boundedness requirement of the leader's state is no more needed.

\section{TRACKING CONSENSUS OF GENERAL NONLINEAR MULTI-AGENT SYSTEMS WITH BOUNDED DISTURBANCES}

In this section, consider the tracking problem of a group of $N+1$ agents described by (1), where the disturbance $d_{i}(t)$ is bounded. i.e.:

Assumption 5: The disturbance of each follower is bounded,

$$
\left\|d_{i}(t)\right\|_{\infty} \leq \omega_{i}, \quad i=1,2, \ldots, N
$$

where $\omega_{i}>0$ are some finite but unknown constants.

First of all, take a further look at the distributed state observer (6). Noticing the special structure of $A^{\prime}=C^{-1}(A+$ $B Q) C, B^{\prime}=C^{-1} B D,(6)$ can be written as

$$
\dot{y}_{i}=A^{\prime} y_{i}+B^{\prime} \hat{u}_{i}+\tau_{i},
$$

where $y_{i}=\left[y_{i 1}, \ldots, y_{i n}\right]^{T}, \hat{u}_{i}=-\left[\left(\alpha_{i 1}+\beta_{i 1}\right)\left[e_{i k_{1}}+\right.\right.$ $\left.\left.\operatorname{sign}\left(e_{i k_{1}}\right)\right], \ldots,\left(\alpha_{i r_{0}}+\beta_{i r_{0}}\right)\left[e_{i k_{r_{0}}}+\operatorname{sign}\left(e_{i k_{r_{0}}}\right)\right], 0, \ldots, 0\right]^{T}$ with $m-r_{0}$ zeros, $\tau_{i}=\left[\tau_{i 1}^{T}, \ldots, \tau_{i r_{0}}^{T}\right]^{T}$, and $\tau_{i, l+1}=$ $-\left[e_{i, k_{l}+1}, \ldots, e_{i, k_{l+1}-1}, 0\right]^{T}, l=0, \ldots, r_{0}-1$. Let $\hat{x}_{i}=C y_{i}$, one has

$$
\dot{\hat{x}}_{i}=A \hat{x}_{i}+B\left(Q \hat{x}_{i}+D \hat{u}_{i}\right)+C \tau_{i} .
$$

Then, consider the dynamics of each follower. Owing to the approximation property of neural networks (NNs) [43], the smooth function $f_{i}\left(x_{i}\right)$ can be approximated on a compact set as

$$
f_{i}\left(x_{i}\right)=W_{i}^{T} \phi_{i}\left(x_{i}\right)+\varepsilon_{i}
$$

where $\phi_{i}(\cdot): R^{n} \rightarrow R^{s}$ is a known activation function, $W_{i} \in R^{s \times m}$ is a constant real matrix representing the ideal $\mathrm{NN}$ weight matrix, and $\varepsilon_{i}$ is the bounded approximation error vector satisfying $\left\|\varepsilon_{i}\right\|_{\infty} \leq \bar{\varepsilon}_{i}$. Usually, $\bar{\varepsilon}_{i}$ is unknown and hence it will not be used in the following controller design. Suppose that $\phi_{i}(\cdot)$ is bounded on compact sets.

For each follower, design the controller as follows:

$$
u_{i}=Q \hat{x}_{i}+D \hat{u}_{i}-\hat{W}_{i} \phi_{i}\left(x_{i}\right)-K \tilde{x}_{i}-\mu_{i} \operatorname{sign}\left(K \tilde{x}_{i}\right),
$$

of which $\hat{W}_{i}$ is the estimation of $W_{i}$ and is utilized to compensate the nonlinearity $f_{i}\left(x_{i}\right), K=B^{T} P$ with $P$ being the solution of (3) $(\bar{\beta}=1), \tilde{x}_{i}=x_{i}-\hat{x}_{i}$, and $\mu_{i}$ is an adaptive gain designed as

$$
\dot{\mu}_{i}=\left\|K \tilde{x}_{i}\right\|_{1}
$$

Furthermore, $\hat{W}_{i}$ is designed as

$$
\begin{aligned}
& \dot{\hat{W}}_{i}=m_{i} \phi_{i}\left(x_{i}\right) \tilde{x}_{i}^{T} P B-m_{i} h_{i}\left(\hat{W}_{i}-\bar{W}_{i}\right), \\
& \dot{\bar{W}}_{i}=n_{i} h_{i}\left(\hat{W}_{i}-\bar{W}_{i}\right),
\end{aligned}
$$

where $m_{i}, n_{i}$, and $h_{i}$ are positive factors.

Theorem 2: Suppose that Assumptions 1-5 are satisfied. Then, with the controller (17)-(19), each follower can track the trajectory of the leader asymptotically.

Proof: Noticing (1), (15), and (17), one obtains the dynamics of $\tilde{x}_{i}$ :

$$
\begin{aligned}
\dot{\tilde{x}}_{i}= & A \tilde{x}_{i}+B\left[\tilde{W}_{i}^{T} \phi_{i}\left(x_{i}\right)+\bar{d}_{i}-K \tilde{x}_{i}-\mu_{i} \operatorname{sign}\left(K \tilde{x}_{i}\right)\right] \\
& -C \tau_{i},
\end{aligned}
$$

where $\tilde{W}_{i}=W_{i}-\hat{W}_{i}$ and $\bar{d}_{i}=\varepsilon_{i}+d_{i}$. Then one has $\left\|\bar{d}_{i}\right\|_{\infty} \leq$ $\omega_{i}+\bar{\varepsilon}_{i}$

Consider the following Lyapunov function candidate

$$
V_{i}=\tilde{x}_{i}^{T} P \tilde{x}_{i}+\frac{1}{m_{i}} \operatorname{tr}\left(\tilde{W}_{i}^{T} \tilde{W}_{i}\right)+\frac{1}{n_{i}} \operatorname{tr}\left(\breve{W}_{i}^{T} \breve{W}_{i}\right)+\left(\mu_{i}-\mu\right)^{2},
$$

of which $\breve{W}_{i}=W_{i}-\bar{W}_{i}, \mu$ is a parameter to be designed. Taking the derivative of $V_{i}$ gives

$$
\begin{aligned}
\dot{V}_{i}= & 2 \tilde{x}_{i}^{T} P\left\{A \tilde{x}_{i}+B\left[\tilde{W}_{i}^{T} \phi_{i}\left(x_{i}\right)+\bar{d}_{i}-K \tilde{x}_{i}-\mu_{i} \operatorname{sign}\left(K \tilde{x}_{i}\right)\right]\right. \\
& \left.-C \tau_{i}\right\}-\frac{2}{m_{i}} \operatorname{tr}\left(\tilde{W}_{i}^{T} \dot{\hat{W}}_{i}\right)-\frac{2}{n_{i}} \operatorname{tr}\left(\breve{W}_{i}^{T} \dot{\bar{W}}_{i}\right)+2\left(\mu_{i}-\mu\right) \dot{\mu}_{i} .
\end{aligned}
$$

On the one hand,

$$
\begin{aligned}
\frac{1}{m_{i}} \operatorname{tr}\left(\tilde{W}_{i}^{T} \dot{\hat{W}}_{i}\right) & =\operatorname{tr}\left(\tilde{W}_{i}^{T} \phi_{i}\left(x_{i}\right) \tilde{x}_{i}^{T} P B-h_{i} \tilde{W}_{i}^{T}\left(\hat{W}_{i}-\bar{W}_{i}\right)\right) \\
& =\tilde{x}_{i}^{T} P B \tilde{W}_{i}^{T} \phi_{i}\left(x_{i}\right)-h_{i} \operatorname{tr}\left(\tilde{W}_{i}^{T}\left(\hat{W}_{i}-\bar{W}_{i}\right)\right) .
\end{aligned}
$$


On the other hand,

$$
\frac{1}{n_{i}} \operatorname{tr}\left(\breve{W}_{i}^{T} \dot{\bar{W}}_{i}\right)=h_{i} \operatorname{tr}\left(\breve{W}_{i}^{T}\left(\hat{W}_{i}-\bar{W}_{i}\right)\right) .
$$

It follows that

$$
\begin{aligned}
\dot{V}_{i}= & \tilde{x}_{i}^{T}\left(P A+A^{T} P-2 P B B^{T} P\right) \tilde{x}_{i}+2 \tilde{x}_{i}^{T} P B \bar{d}_{i} \\
& -2 \mu\left\|K \tilde{x}_{i}\right\|_{1}-2 \tilde{x}_{i}^{T} P C \tau_{i} \\
& -2 h_{i} \operatorname{tr}\left(\left(\hat{W}_{i}-\bar{W}_{i}\right)^{T}\left(\hat{W}_{i}-\bar{W}_{i}\right)\right) \\
\leq & -\left\|\tilde{x}_{i}\right\|^{2}+2\left(\omega_{i}+\bar{\varepsilon}_{i}-\mu\right)\left\|K \tilde{x}_{i}\right\|_{1}-2 \tilde{x}_{i}^{T} P C \tau_{i},
\end{aligned}
$$

where $\tilde{x}_{i}^{T} P B \operatorname{sign}\left(K \tilde{x}_{i}\right)=\left\|K \tilde{x}_{i}\right\|_{1}$ and $\tilde{W}_{i}-\breve{W}_{i}=-\hat{W}_{i}+\bar{W}_{i}$ are applied in the first equality. Choose $\mu>\omega_{i}+\bar{\varepsilon}_{i}$. Noticing that $-2 \tilde{x}_{i}^{T} P C \tau_{i} \leq \varphi\left\|\tilde{x}_{i}\right\|^{2}+\frac{1}{\varphi}\left\|P C \tau_{i}\right\|^{2}$ with $0<\varphi<1$, one further has

$$
\dot{V}_{i} \leq-(1-\varphi)\left\|\tilde{x}_{i}\right\|^{2}+\frac{1}{\varphi}\left\|P C \tau_{i}\right\|^{2} .
$$

According to Theorem $1, \tau_{i} \rightarrow 0$ as $t \rightarrow \infty$. Observing from (11), one can also obtain that $\dot{V}_{o} \leq$ $-\frac{\lambda_{0}}{2} \sum_{i=1}^{N} \alpha_{i 1}(0)\left\|e_{i p_{1}}\right\|^{2}$, which follows that $\int_{0}^{\infty}\left\|e_{i p_{1}}\right\|^{2} d t$ exists and is finite. Furthermore, one can conclude from (8) that $\int_{0}^{\infty}\left\|e_{i 1}\right\|^{2} d t, \ldots, \int_{0}^{\infty}\left\|e_{i, p_{1}-1}\right\|^{2} d t$ exist and are finite. With similar arguments, one can claim that $\int_{0}^{\infty}\left\|e_{i 1}\right\|^{2} d t, \ldots, \int_{0}^{\infty}\left\|e_{i, n}\right\|^{2} d t$ exist and are finite. Noticing the definition of $\tau_{i}$, one can further get that $\int_{0}^{\infty}\left\|P C \tau_{i}\right\|^{2} d t$ exists and is finite. It follows that $V_{i}$ is bounded and $\int_{0}^{\infty}\left\|\tilde{x}_{i}\right\|^{2} d t$ exists and is finite. By invoking (21), $\tilde{x}_{i}, \tilde{W}_{i}$, $\mu_{i}$ are bounded. Then $\tilde{x}_{i}^{T} \dot{\tilde{x}}_{i}$ is bounded by noticing (20). According to Barbalat Lemma [46], one has $\tilde{x}_{i} \rightarrow 0$, as $t \rightarrow \infty$. This completes the proof.

Remark 5: Inspired by [44] and [45], the so-called $\sigma$-modification method can be applied for the adaptive gain $\mu_{i}$ to avoid high amplitude. Specifically, (18) can be modified as:

$$
\begin{aligned}
& \dot{\mu}_{i}=\left\|K \tilde{x}_{i}\right\|_{1}-\bar{h}_{i}\left(\mu_{i}-\hat{\mu}_{i}\right), \\
& \dot{\hat{\mu}}_{i}=\bar{h}_{i}\left(\mu_{i}-\hat{\mu}_{i}\right),
\end{aligned}
$$

where $\bar{h}_{i}$ is a positive factor. In fact, the design of (19) has similar effects. Different from the work in [44], the upper bound of the neural network approximation error as well as the disturbance is not required to be known.

\section{TRACKING CONSENSUS OF GENERAL NONLINEAR MULTI-AGENT SYSTEMS WITH DISTURBANCES GENERATED BY EXOSYSTEM}

In this section, consider the tracking problem of a group of $N+1$ agents described by (1), and the disturbance $d_{i}(t)$ is generated by an exosystem:

$$
\left\{\begin{array}{l}
\dot{\xi}_{i}=W \xi_{i}, \\
d_{i}=V \xi_{i},
\end{array}\right.
$$

of which $\xi_{i}$ is the state of the exosystem (24), $W$ and $V$ are with appropriate dimensions.

Assumption 6: The pair $(B V, W)$ is observable.

In the last section, the general smooth nonlinearity was considered. In this section, another class of nonlinearity will be discussed.

Assumption 7: Assume that $f_{i}\left(x_{i}\right)=H_{i}^{T} v_{i}\left(x_{i}\right)$, where $H_{i} \in R^{s \times m}$ is the unknown constant coefficient matrix, and $v_{i}(\cdot): R^{n} \rightarrow R^{s}$ is a known bounded function.
In the following, a class of disturbance-observer-based tracking control protocols is proposed to solve the tracking problem:

$$
\begin{aligned}
\dot{z}_{i}= & (W+F B V)\left(z_{i}-F x_{i}\right)+F A x_{i}+F B u_{i} \\
& +F B \hat{H}_{i}^{T} v_{i}\left(x_{i}\right) \\
\hat{d}_{i}= & V \hat{\xi}_{i}, \hat{\xi}_{i}=z_{i}-F x_{i} \\
u_{i}= & Q \hat{x}_{i}+D \hat{u}_{i}-\hat{H}_{i}^{T} v_{i}\left(x_{i}\right)-K \tilde{x}_{i}-\hat{d}_{i}
\end{aligned}
$$

of which $z_{i}$ and $\hat{d}_{i}$ are the state and output of the disturbance observer for (24), $F$ is chosen such that $W+F B V$ is Hurwitz, $K=B^{T} P$ with $P$ being the solution of $(3)(\bar{\beta}=1), \tilde{x}_{i}, \hat{u}_{i}$ and $\hat{x}_{i}$ are defined the same as the previous section. Assumption 6 ensures the existence of $F$. Furthermore, $\hat{H}_{i}$ is the estimation of $H_{i}$ with the following dynamics:

$$
\dot{\hat{H}}_{i}=g_{i} v_{i}\left(x_{i}\right) \tilde{x}_{i}^{T} P B-g_{i} \epsilon_{i} \hat{H}_{i},
$$

of which $g_{i}$ and $\epsilon_{i}$ are positive factors.

Theorem 3: Suppose that Assumptions 1-4, 6 and 7 are satisfied. Then with the controller design (25) and (26), each follower can track to a bounded neighborhood of the leader's state.

Proof: Denote $e_{\xi i}=\xi_{i}-\hat{\xi}_{i}$, and $\tilde{H}_{i}=H_{i}-\hat{H}_{i}$. By invoking (15), (24), and (25), one can obtain the dynamics of $e_{\xi i}$ and $\tilde{x}_{i}$ :

$$
\begin{aligned}
\dot{e}_{\xi i} & =(W+F B V) e_{\xi i}+F B \tilde{H}_{i}^{T} v_{i}\left(x_{i}\right) \\
\dot{\tilde{x}}_{i} & =A \tilde{x}_{i}+B \tilde{H}_{i}^{T} v_{i}\left(x_{i}\right)-B K \tilde{x}_{i}+B V e_{\xi i}-C \tau_{i} .
\end{aligned}
$$

Consider the following Lyapunov function candidate

$$
V_{e i}=\tilde{x}_{i}^{T} P \tilde{x}_{i}+\frac{1}{g_{i}} \operatorname{tr}\left(\tilde{H}_{i}^{T} \tilde{H}_{i}\right)+\lambda e_{\xi i}^{T} P_{1} e_{\xi_{i}},
$$

where $P_{1}$ is a positive definite matrix satisfying $X=(W+$ $F B V)^{T} P_{1}+P_{1}(W+F B V)<0$ and $\lambda>0$ is a parameter to be designed.

Taking the derivative of $V_{e i}$, one has

$$
\begin{aligned}
\dot{V}_{e i}= & \tilde{x}_{i}^{T}\left(P A+A^{T} P-2 P B B^{T} P\right) \tilde{x}_{i} \\
& +2 \tilde{x}_{i}^{T} P B V e_{\xi i}-2 \tilde{x}_{i}^{T} P C \tau_{i}+2 \epsilon_{i} \operatorname{tr}\left(\tilde{H}_{i}^{T} \hat{H}_{i}\right) \\
& +\lambda e_{\xi i}^{T} X e_{\xi i}+2 \lambda e_{\xi i}^{T} P_{1} F B \tilde{H}_{i}^{T} v_{i}\left(x_{i}\right) .
\end{aligned}
$$

Noticing that $\left\|\tilde{H}_{i}\right\|_{F}^{2}=\operatorname{tr}\left(\tilde{H}_{i}^{T} \tilde{H}_{i}\right)$, one has

$$
\begin{aligned}
2 \tilde{x}_{i}^{T} P B V e_{\xi i} & \leq \frac{1}{4}\left\|\tilde{x}_{i}\right\|^{2}+4 \eta_{1}\left\|e_{\xi i}\right\|^{2}, \\
2 \tilde{x}_{i}^{T} P C \tau_{i} \leq & \frac{1}{4}\left\|\tilde{x}_{i}\right\|^{2}+4\left\|P C \tau_{i}\right\|^{2}, \\
2 \epsilon_{i} \operatorname{tr}\left(\tilde{H}_{i}^{T} \hat{H}_{i}\right)= & -2 \epsilon_{i} \operatorname{tr}\left(\tilde{H}_{i}^{T} \tilde{H}_{i}\right)+2 \epsilon_{i} \operatorname{tr}\left(\tilde{H}_{i}^{T} H_{i}\right) \\
& \leq-\epsilon_{i}\left\|\tilde{H}_{i}\right\|_{F}^{2}+\epsilon_{i}\left\|H_{i}\right\|_{F}^{2}, \\
e_{\xi i}^{T} X e_{\xi i} \leq & -\eta_{2}\left\|e_{\xi i}\right\|^{2}, \\
2 e_{\xi i}^{T} P_{1} F B \tilde{H}_{i}^{T} v_{i}\left(x_{i}\right) \leq & \frac{2}{\eta_{2}}\left\|v_{i}\left(x_{i}\right)\right\|^{2}\left\|P_{1} F B\right\|^{2}\left\|\tilde{H}_{i}\right\|^{2} \\
& +\frac{\eta_{2}}{2}\left\|e_{\xi i}\right\|^{2} \\
\leq & \frac{\eta_{2}}{2}\left\|e_{\xi i}\right\|^{2}+\eta_{3}\left\|\tilde{H}_{i}\right\|_{F}^{2},
\end{aligned}
$$

where $\eta_{1}=\lambda_{\max }\left(V^{T} B^{T} P^{2} B V\right), \eta_{2}=\lambda_{\min }(-X), \eta_{3}=$ $\frac{\eta_{2}}{2}\left\|P_{1} F B\right\|^{2} \bar{v}$, and $\bar{v}$ is the upper bound of $\left\|v_{i}\left(x_{i}\right)\right\|^{2}$. Choose 
$\lambda=\left(8 \eta_{1}+2 \delta\right) / \eta_{2}, \epsilon_{i}=\lambda \eta_{3}+\bar{\delta}$, where $\delta$ and $\bar{\delta}$ are two positive numbers. It follows that

$$
\begin{aligned}
\dot{V}_{e i} & \leq-\frac{1}{2}\left\|\tilde{x}_{i}\right\|^{2}-\delta\left\|e_{\xi i}\right\|^{2}-\bar{\delta}\left\|\tilde{H}_{i}\right\|_{F}^{2}+\theta \\
& \leq-\kappa V_{e i}+\theta
\end{aligned}
$$

of which $\kappa=\frac{\min \{1 / 2, \delta, \bar{\delta}\}}{\max \left\{\lambda_{\max }(P), 1 / g_{i}, \lambda \cdot \lambda_{\max }\left(P_{1}\right)\right\}}$ and $\theta=$ $4\left\|P C \tau_{i}\right\|^{2}+\epsilon_{i}\left\|H_{i}\right\|_{F}^{2}$. Since $\tau_{i} \rightarrow 0$ as $t \rightarrow \infty$. It follows from (30) that $\tilde{x}_{i}$ will converge to the compact set $\Omega=\left\{\tilde{x}_{i}\right.$ : $\left.V_{e i} \leq \frac{\bar{\theta}}{\kappa}\right\}$, where $\bar{\theta}=\epsilon_{i}\left\|H_{i}\right\|_{F}^{2}$. This completes the proof.

\section{Simulation ExAmples}

In this section, two simulations are provided to verify the theoretical results. Consider that there are 5 agents with 1 leader labeled as 0 , and 4 followers labeled from 1 to 4 . The directed communication topology is depicted in Fig. 1 with all the weights indicated on the communication edges. The corresponding Laplacian matrix is $L=\left(\begin{array}{ccccc}0 & 0 & 0 & 0 & 0 \\ -2 & 2 & 0 & 0 & 0 \\ 0 & -1.5 & 1.5 & 0 & 0 \\ 0 & 0 & -1.5 & 1.5 & 0 \\ -2 & 0 & 0 & 0 & 2\end{array}\right)$, and $\bar{L}=$ $\left(\begin{array}{cccc}2 & 0 & 0 & 0 \\ -1.5 & 1.5 & 0 & 0 \\ 0 & -1.5 & 1.5 & 0 \\ 0 & 0 & 0 & 2\end{array}\right)$. For each agent, denote $x_{i}=$
$\left[\begin{array}{l}x_{i 1} \\ x_{i 2} \\ x_{i 3}\end{array}\right], i=0,1, \ldots, 4$. Take $A=\left[\begin{array}{lll}0 & 1 & 0 \\ 0 & 0 & 1 \\ 0 & 0 & 0\end{array}\right]$, and $B=\left[\begin{array}{l}0 \\ 0 \\ 1\end{array}\right]$. In both examples, the leader's input is chosen as $u_{0}(t)=$ $\tanh \left(x_{01}+2 x_{02}\right)+\sin \left(x_{03}-1\right)$. The initial values of the agents' states are generated randomly.

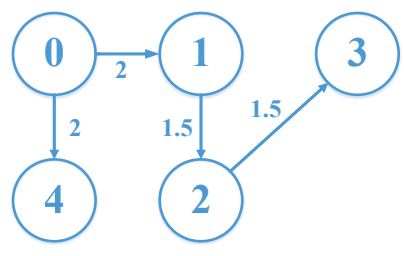

Fig. 1. Communication topology of 1 leader and 4 followers.

\section{A. Example 1: followers with bounded disturbance}

In this example, the followers are assumed to be influenced by bounded disturbances and general nonlinear dynamics:

$$
\begin{aligned}
f_{i}\left(x_{i}\right) & =x_{i 1} \cdot \cos \left(x_{i 2}\right)+x_{i 2} \cos \left(x_{i 3}\right), \\
d_{i}(t) & =\sin \left(x_{i 1}+x_{i 3}\right)+2 \cos \left(1+x_{i 2}\right) .
\end{aligned}
$$

The distributed observer and the tracking controller are given in (6) and (17), respectively. The adaptive gain $\alpha_{i}$ is designed according to Remark 3, and the adaptive control gain $\mu_{i}$ is designed according to Remark 5 with $\bar{h}_{i}=1$. It is easy to see that $r_{0}=1$. Hence, randomly set the initial value of the adaptive gain such that $\alpha_{i}(0)>0$. In the simulation, a onelayer NN with 50 neurons is used for each follower. Sigmoid basis function $\sigma(z)=\left(1+e^{-z}\right)^{-1}$ is employed and the NN weights $\hat{W}_{i}$ are initialized as zero. Besides, the parameters in (19) are chosen as $m_{i}=1000, h_{i}=0.1, n_{i}=20$. The initial states of each agent and observer are generated randomly. Furthermore, solving the LMI (3) with $\beta=1$ by using LMI toolbox of Matlab gives $P=\left[\begin{array}{ccc}5.1201 & 5.7017 & 2.1169 \\ 5.7017 & 10.4176 & 4.3302 \\ 2.1169 & 4.3302 & 3.0545\end{array}\right]$. It follows that $K=B^{T} P=[2.1169,4.3302,3.0545]$.

Fig. 2 depicts the estimation error between the distributed observer and the leader, which shows that the proposed distributed observer has good performance. Fig. 3 shows the tracking error among each follower and the leader. One can see that with the observer based controller (17), each follower can track the leader's state eventually. Figs. 4 and 5 depict the evolution of the adaptive gains $\alpha_{i}$ and $\mu_{i}$.

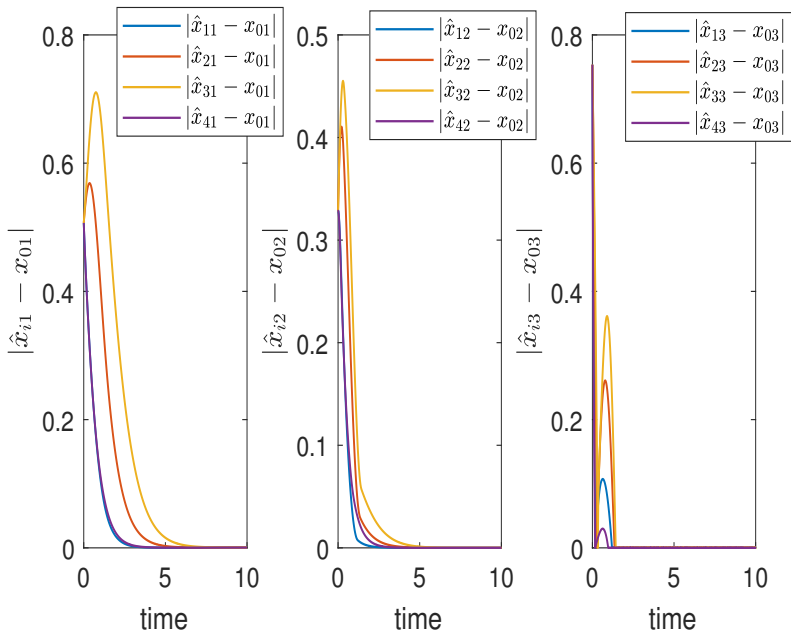

Fig. 2. Example 1: The trajectories of the estimation error $\hat{x}_{i}-x_{0}, i=$ $1, \ldots, 4$.
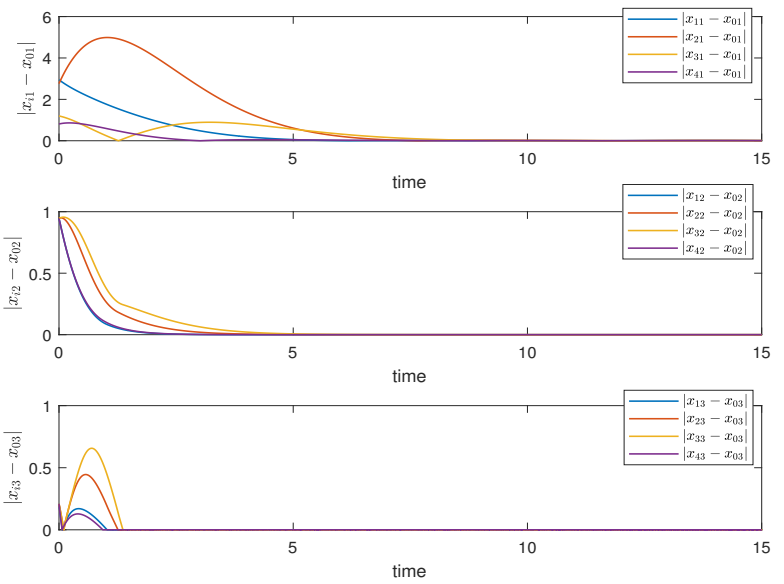

Fig. 3. Example 1: Trajectories of the tracking error $x_{i}-x_{0}, i=1, \ldots, 4$.

B. Example 2: followers with disturbance generated by exosystems

In this example, each follower is assumed to be influenced by heterogeneous nonlinearity and external disturbances (24), 


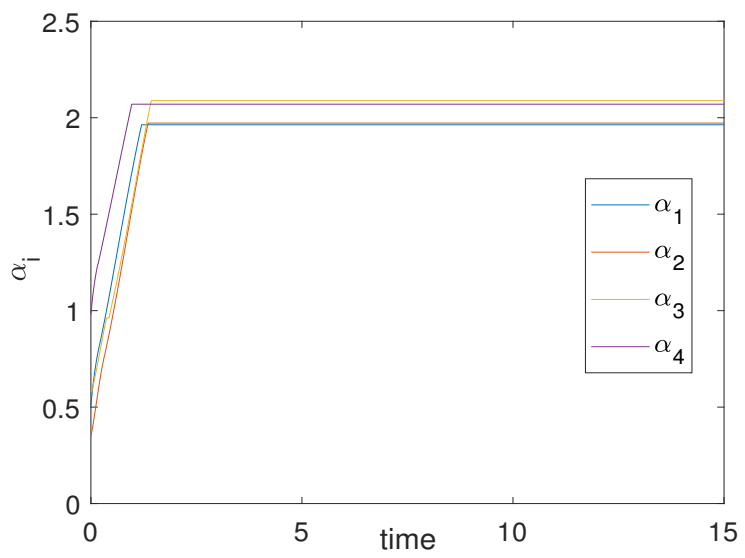

Fig. 4. Example 1: Evolution of the adaptive gain $\alpha_{i}, i=1, \ldots, 4$.

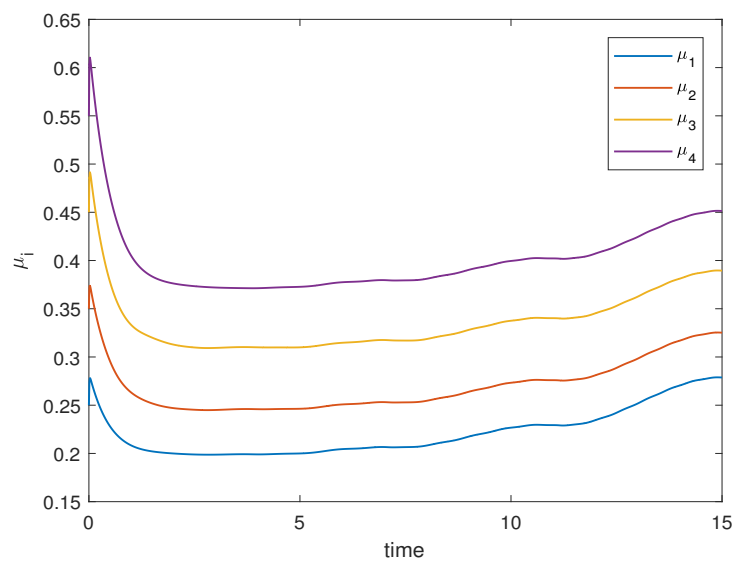

Fig. 5. Example 1: Evolution of the adaptive gain $\mu_{i}, i=1, \ldots, 4$.

where

$$
\begin{aligned}
H_{i} & =[i / 2, i-1 / 2,3]^{T}, \\
v_{i}\left(x_{i}\right) & =\left[\sin \left(x_{i 1} * x_{i 3}\right), \tanh \left(x_{i 1}-x_{i 3}\right), \cos \left(x_{i 3}\right)+1\right]^{T}, \\
W & =\left[\begin{array}{lll}
0 & 1 & 1 \\
0 & 1 & 0 \\
1 & 0 & 0
\end{array}\right], V=[0,0,1] .
\end{aligned}
$$

The observer-based control protocol is designed as (25). Choose $F=\left[\begin{array}{ccc}0 & 0 & -19 \\ 0 & 0 & -24 \\ 0 & 0 & -7\end{array}\right]$. solving LMI $X=(W+$ $F B V)^{T} P_{1}+P_{1}(W+F B V)<0$ gives $P_{1}=$ $\left[\begin{array}{lll}3.3160 & 3.9092 & 0.2766\end{array}\right]$

$\begin{array}{lll}3.9092 & 8.0513 & 0.4082\end{array}$.

$\left[\begin{array}{lll}0.2766 & 0.4082 & 0.1137\end{array}\right]$

The tracking error for each follower is described in Fig. 6 . One can observe that each follower can track to a bounded neighbor of the leader eventually.

\section{CONCLUSION}

In this paper, the tracking consensus problem of general nonlinear multi-agent systems with external disturbances has been discussed. The leader is assumed to have bounded
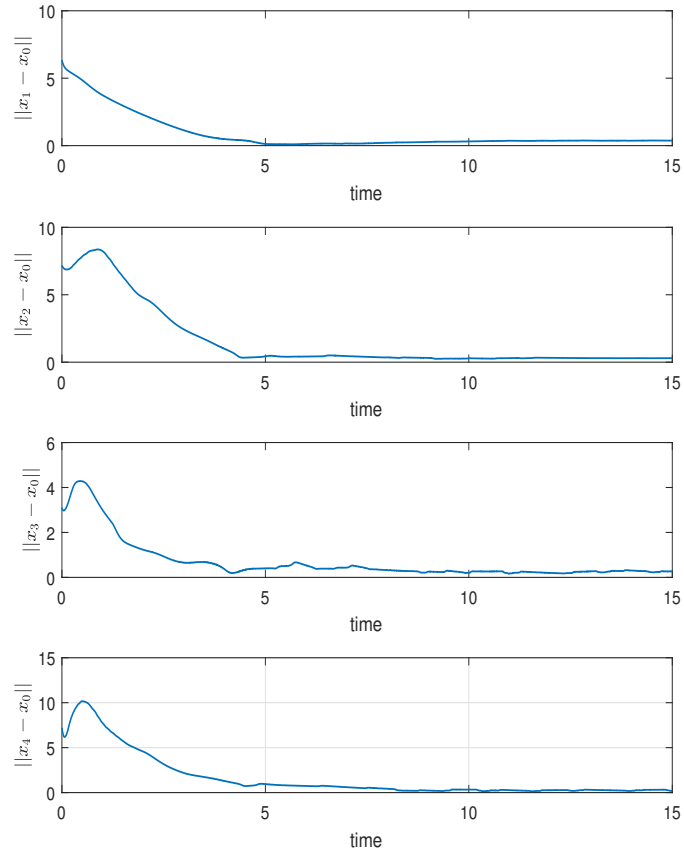

Fig. 6. Example 2: Evolution of the tracking error of each follower.

input. A new class of fully distributed state observer for the leader has been proposed under the assumption that the upper bound of the leader's input is unknown to all the followers. Furthermore, two distributed observer-based control protocols have been designed for solving the tracking problems where each follower may be influenced by bounded disturbances (the upper bound is assumed unknown) or disturbances generated by exosystems, respectively. Neural networks and adaptive laws have been applied for each follower to compensate their own nonlinearity. One further work is to consider the fully distributed problems with both general nonlinearity and mismatched disturbances under directed communication topologies.

\section{REFERENCES}

[1] D. Fox, W. Burgard, H. Kruppa, and S. Thrun, "A probabilistic approach to collaborative multi-robot localization," Auton. Robots, vol. 8, no. 3, pp. $325-344,2000$.

[2] M. Campbell, "Planning algorithm for multiple satellite clusters," $J$. Guidance Control Dynam., vol. 26, no. 5, pp. 770-780, 2003.

[3] R. Beard, T. McLain, M. Goodrich, and E. Anderson, "Coordinated target assignment and intercept for unmanned air vehicles," IEEE Robotic. Autom., vol. 18, no. 6, pp. 911-922, 2002.

[4] L. Eschenauer and V. Gligor, "A key-management scheme for distributed sensor networks," in Proc. ACM CCS, 2002, pp. 41-47.

[5] R. Olfati-Saber and R. Murray, "Consensus problems in networks of agents with switching topology and time-delays," IEEE Trans. Autom. Control, vol. 49, no. 9, pp. 1520-1533, 2004.

[6] A. Fax and R. M. Murray, "Information flow and cooperative control of vehicle formations," IEEE Trans. Autom. Control, vol. 49, no. 9, pp. 1465-1476, 2004.

[7] Z. Qu, Cooperative Control of Dynamical Systems: Applications to Autonomous Vehicles. London, U.K.: Springer-Verlag, 2009.

[8] C. Li, X. Yu, T. Huang, and X. He, "Distributed optimal consensus over resource allocation network and its application to dynamical economic dispatch," IEEE Trans. Neural Netw. Learn. Syst., vol. 29, no. 6, pp. 2407-2418, 2018. 
[9] Y. Hong, J. Hu, and L. Gao, "Tracking control for multi-agent consensus with an active leader and variable topology," Automatica, vol. 42, no. 7, pp. 1177-1182, 2006.

[10] W. Ren and R. W. Beard, "Consensus seeking in multiagent systems under dynamically changing interaction topologies," IEEE Trans. Autom. Control, vol. 50, no. 5, pp. 655-661, 2005.

[11] Z. Ding, "Consensus disturbance rejection with disturbance observers," IEEE Trans. Ind. Electron., vol. 62, no. 9, pp. 5829-5837, 2015.

[12] W. Yu, G. Wen, G. Chen, and J. Cao, Distributed Cooperative Control of Multi-agent Systems, Wiley\&Higher Education Press, 2016.

[13] W. Yu, G. Chen, and M. Cao, "Some necessary and sufficient conditions for second-order consensus in multi-agent dynamical systems," Automatica, vol. 46, no. 6, pp. 1089-1095, 2010.

[14] W. Yu, G. Chen, M. Cao, and J. Kurths, "Second-order consensus for multi-agent systems with directed topologies and nonlinear dynamics," IEEE Trans. Syst., Man, Cybern., Cybern., vol. 40, no. 3, pp. 881-891, 2010.

[15] W. Yu, H. Wang, F. Cheng, X. Yu, and G. Wen, "Second-order consensus in multiagent systems via distributed sliding mode control," IEEE Trans. Cybern., vol. 47, no. 8, pp. 1872-1881, 2017.

[16] S. Yu and X. Long, "Finite-time consensus for second-order multi-agent systems with disturbances by integral sliding mode," Automatica, vol. 54, no. 4, pp. 158-165, 2015.

[17] Z. Guan, F. Sun, Y. Wang, and T. Li, "Finite-time consensus for leaderfollowing second-order multi-agent networks," IEEE Trans. Circuits Syst. I. Regul. Pap., vol. 59, no. 11, pp. 2646-2654, 2012.

[18] H. Hong, W. Yu, X. Yu, G. Wen, and A. Alsaedi, "Fixed-time connectivity-preserving distributed average tracking for multi-agent systems," IEEE Trans. Circuits Syst. II, Exp. Briefs, vol. 64, no. 10, pp. 1192-1196, 2017.

[19] M. Franceschelli, A. Pisano, A. Giua, and E. Usai, "Finite-time consensus with disturbance rejection by discontinuous local interactions in directed graphs," IEEE Trans. Autom. Control, vol. 60, no. 4, pp. 1133$1138,2015$.

[20] H. Hong, W. Yu, G. Wen, and X. Yu, "Distributed robust fixed-time consensus for nonlinear and disturbed multiagent systems," IEEE Trans. Syst., Man, Cybern., Syst., vol. 47, no. 7, pp. 1464-1473, 2017.

[21] W. Ren and R. W. Beard, "Consensus seeking in multiagent systems under dynamically changing interaction topologies," IEEE Trans. Autom. Control, vol. 50, no. 5, pp. 655-661, 2005.

[22] C. Altafini, "Consensus problems on networks with antagonistic interactions," IEEE Trans. Autom. Control, vol. 58, no. 4, pp. 935-946, 2013.

[23] X. Wang and Y. Hong, "Finite-time consensus for multi-agent networks with second-order agent dynamics," in Proc. the 17th World Congress, IFAC, Korea: Seoul, pp. 15185-15190, Jul. 2008.

[24] W. Yu, G. Chen, W. Ren, J. Kurths, and W. Zheng, "Distributed higher order consensus protocols in multiagent dynamical systems," IEEE Trans. Circuits Syst. I. Regul. Pap., vol. 58, no. 8, pp. 1924-1932, 2011.

[25] W. Ren, K. Moore, and Y. Chen, "High-order and model reference consensus algorithms in cooperative control of multivehicle systems," J. Dynam. Systems Measurement Control, vol. 129, no. 5, pp. 678-688, 2007.

[26] J. Fu and J. Wang, "Adaptive consensus tracking of high-order nonlinear multi-agent systems with directed communication graphs," Internat. J. Control, Autom. and Systems, vol. 12, no. 5, pp. 919-929, 2014.

[27] S. Khoo, L. Xie, S. Zhao, and Z. Man, "Multi-surface sliding control for fast finite-time leader-follower consensus with high order SISO uncertain nonlinear agents," Internat. J. Robust Nonlinear Control, vol. 24, no. 16, pp. 2388-2404, 2014.

[28] C. Ma and J. Zhang, "Necessary and sufficient conditions for consensusability of linear multi-agent systems," IEEE Trans. Autom. Control, vol. 55, no. 5, pp. 1263-1268, 2010.

[29] G. Wen, Z. Li, Z. Duan, and G. Chen, "Distributed consensus control for linear multi-agent systems with discontinuous observations," Internat. J. Control, vol. 86, no. 1, pp. 95-106, 2013.

[30] Z. Li, X. Liu, W. Ren, and L. Xie, "Distributed tracking control for linear multiagent systems with a leader of bounded unknown input," IEEE Trans. Autom. Control, vol. 58, no. 2, pp. 518-523, 2013.

[31] Z. Li, G. Wen, Z. Duan, and W. Ren, "Designing fully distributed consensus protocols for linear multi-agent systems with directed graphs," IEEE Trans. Autom. Control, vol. 60, no. 4, pp. 1152-1157, 2015.

[32] Y. Lü, Z. Li, and Z. Duan, "Distributed adaptive consensus protocols for linear multi-agent systems with directed graphs and a leader of unknown control input," in Proc. 34th Chinese Control Conf., 2015, pp. 73397343.
[33] Y. Lü, Z. Li, Z. Duan, and J. Chen, "Distributed adaptive output feedback consensus protocols for linear systems on directed graphs with a leader of bounded input," Automatica, vol. 74, no. 12, pp. 308-314, 2016.

[34] Z. Li, and Z. Duan, "Distributed tracking control of multi-agent systems with heterogeneous uncertainties," in Proc. 10th IEEE Int. Conf. Control Autom., 2013, pp. 1956-1961.

[35] G. Wen, Y. Zhao, Z. Duan, W. Yu, and G. Chen, "Containment of higherorder multi-leader multi-agent systems: a dynamic output approach," IEEE Trans. Autom. Control, vol. 61, no. 4, pp. 1135-1140, 2016.

[36] Z. Peng, D. Wang, H. Zhang, and Y. Lin, "Cooperative output feedback adaptive control of uncertain nonlinear multi-agent systems with a dynamic leader," Neurocomputing, vol. 149, Part A, pp. 132-141, 2015.

[37] J. Sun, Z. Geng, and Y. Lü, "Adaptive output feedback consensus tracking for heterogeneous multi-agent systems with unknown dynamics under directed graphs," Systems Control Lett., vol. 87, no. 1, pp. 16-22, 2016.

[38] Z. Ding and Z. Li, "Distributed adaptive consensus control of nonlinear output-feedback systems on directed graphs," Automatica, vol. 72, no. 10, pp. 46-52, 2016.

[39] G. Wen, W. Yu, Y. Xia, X. Yu, and J. Hu, "Distributed tracking of nonlinear multi-agent systems under directed switching topology: An observer-based protocol," IEEE Trans. Syst., Man, Cybern., Syst., vol. 47 , no. 5 , pp. $869-881,2017$.

[40] W. Xu, D. Ho, L. Li, and J. Cao, "Event-triggered schemes on leaderfollowing consensus of general linear multiagent systems under different topologies," IEEE Trans. Cybern., vol. 47, no. 1, pp. 212-223, 2017.

[41] R. A. Horn and C. R. Johnson, Matrix Analysis, Cambridge, U.K.: Cambridge Univ. Press, 1990.

[42] P. Brunovský, "A classification of linear controllable systems," Kybernetika, vol. 6, no. 3 pp. 173-188, 1970.

[43] F. L. Lewis, S. Jagannathan, and A. Yesildirek, Neural Network Control of Robot Manipulators and Non-Linear Systems, New York: Taylor \& Francis, 1998

[44] G. Wen, P. Wang, T. Huang, W. Yu, and J. Sun, "Robust neuroadaptive containment of multileader multiagent systems with uncertain dynamics," IEEE Trans. Syst., Man, Cybern., Syst., to be published, doi: 10.1109/TSMC.2017.2722042.

[45] J. Mei, W. Ren, and J. Chen, "Distributed consensus of second-order multi-agent systems with heterogeneous unknown inertias and control gains under a directed graph," IEEE Trans. Autom. Control, vol. 61, no. 8, pp. 2019-2034, 2016.

[46] H. K. Khalil, Nonlinear Systems, 3rd ed. Upper Saddle River, NJ, USA: Prentice-Hall, 2002.

[47] P. L. Lancaster and Rodman, Algebraic Riccati Equations. London, U.K.: Oxford Univ. Press, 1995. 